\title{
El síndrome metabólico: De factor agravante a principal factor de riesgo patogénico en diversas enfermedades crónicas
}

\author{
ROMMY VON BERNHARDI ${ }^{1}$, SILVANA ZANLUNGO ${ }^{2 a}$, MARCO ARRESE ${ }^{2}$, \\ ANTONIO ARTEAGA ${ }^{3}$, ATTILIO RIGOTTI ${ }^{2}$
}

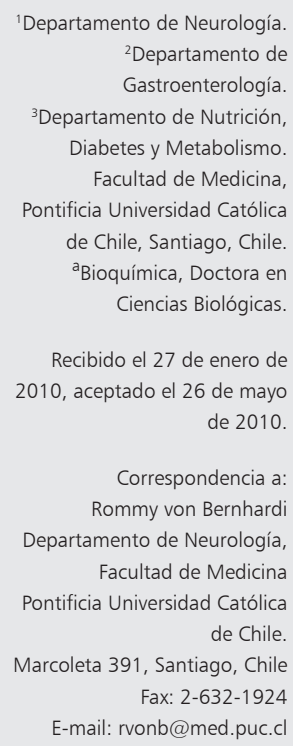
ntre los factores que determinan un mayor riesgo para el desarrollo futuro de enfermedades crónicas, existe un conjunto de alteraciones físicas y metabólicas, incluyendo obesidad central, presión sanguínea elevada, resistencia a la insulina y dislipidemia, que presentan una alta frecuencia de asociación clínica en pacientes, constituyendo una agrupación sindromática característica (Tabla 1). Esta agrupación dio origen al concepto clínico denominado "síndrome metabólico" (SMetab), reconocido por un grupo importante de investigadores, pero aso- ciado también a una gran polémica en el mundo científico ${ }^{1}$. El SMetab tiene una prevalencia muy elevada, superior a $20 \%$ de la población adulta ${ }^{2}$ en el mundo ${ }^{3}$. Existen varios criterios que definen el SMetab, propuestos por la World Health Organization $\left(\mathrm{WHO}^{4}\right)$, el National Cholesterol Education Program Adult Treatment Panel III $^{5}$ y la International Diabetes Federation ${ }^{3}$. El criterio más utilizado es el propuesto por el NCEP ATP III, que considera la presencia de al menos 3 elementos para el diagnóstico (Tabla 1), aunque recientemente se propuso una definición y criterios diagnósticos armonizados entre varias de las organizaciones mencionadas ${ }^{6}$. 
Tabla 1. Criterios diagnósticos del Síndrome Metabólico

\begin{tabular}{|c|c|}
\hline Factor & Criterio* \\
\hline 1 & $\begin{array}{l}\text { Cintura }>88 \mathrm{~cm} \text { (mujeres) } 0>102 \mathrm{~cm} \\
\text { (hombres) }\end{array}$ \\
\hline 2 & $\begin{array}{l}\mathrm{HDL}-\mathrm{C}<50 \mathrm{mg} / \mathrm{dl} \text { (mujeres) o }<40 \mathrm{mg} / \mathrm{dl} \\
\text { (hombres) }\end{array}$ \\
\hline 3 & $\begin{array}{l}\text { Triglicéridos en ayuno }>150 \mathrm{mg} / \mathrm{dl}(1,69 \\
\mathrm{nmol} / \mathrm{L})\end{array}$ \\
\hline 4 & Presión sanguínea > 130/85mmHg \\
\hline 5 & $\begin{array}{l}\text { Glucosa en ayuno }>110 \mathrm{mg} / \mathrm{dl}(>6,1 \\
\mathrm{mmol} / \mathrm{L})\end{array}$ \\
\hline
\end{tabular}

* El diagnóstico requiere la presencia de 3 o más de estos criterios.

\section{Síndrome metabólico en Chile}

La población chilena actualmente tiene una expectativa de vida alta, pero presenta una alta tasa de factores de riesgo predisponentes al desarrollo de enfermedades crónicas, las que muestran una prevalencia elevada en el adulto ${ }^{7}$. Se plantea que este perfil de riesgo es consecuencia de los cambios epidemiológicos y de salubridad que han reducido muchas enfermedades infectocontagiosas, aumentando la incidencia relativa de enfermedades crónicas no transmisibles, y de cambios de hábitos y estilos de vida que aumentan el riesgo de aparición y la gravedad de estas enfermedades crónicas. El fenómeno no es propio de Chile. Se calcula que para el año 2025, la prevalencia mundial de enfermedades crónicas no transmisibles se habrá duplicado, con la mitad de los afectados residiendo en regiones de ingresos per capita bajos o medios ${ }^{7}$. En Chile, una muestra transversal evaluada en la Encuesta Nacional de Salud 2003, mostró una prevalencia de SMetab de 23\% (aproximadamente 2,7 millones de chilenos), similar a la informada en países desarrollados ${ }^{3}$. Un re-análisis de esta encuesta con los criterios actualizados propuestos por NCEP ATP III e IDF ${ }^{8}$, indica que la prevalencia de SMetab en Chile se eleva a 32\% y 37\%, respectivamente, con un nivel de concordancia de $90 \%$ para ambos criterios, mucho mayor que la descrita en otros países ${ }^{9}$.

Entre las enfermedades crónicas más prevalentes, la enfermedad cardiovascular (ECV) ateroesclerótica y la diabetes tipo 2 han sido identificadas como las consecuencias clínicas principales del $\mathrm{SMetab}^{10}$. Sin embargo, individuos con SMetab también son más susceptibles a otras patologías crónicas, como el hígado graso, la litiasis biliar, el asma, y algunas formas de cáncer. Además, evidencias recientes sugieren que el SMetab también impacta sobre el desarrollo de procesos neurodegenerativos, entre los que tienen especial significado la enfermedad de Alzheimer ${ }^{11,62}$. Por todo ello, el SMetab y sus complicaciones a largo plazo podrían convertirse en una carga socioeconómica y de salud pública dramática para países en vías de desarrollo como Chile ${ }^{12}$.

\section{Fisiopatología del síndrome metabólico}

La fisiopatología básica del SMetab es objeto de controversia e investigación activa ${ }^{1}$. Aunque todavía no está claramente definido, el SMetab, más allá del efecto directo e inmediato de la obesidad central, la hiperglicemia, la dislipidemia y la hipertensión por separado, sería una condición fisiopatológica diferente al hiperinsulinismo, capaz de actuar de manera sinérgica a través de la asociación y potenciación de mecanismos biológicos involucrados en la génesis y evolución de diversas enfermedades crónicas en adición a la patología cardiovascular y diabetes ${ }^{13}$.

El SMetab es conceptualmente complejo, tanto por los procesos fisiopatológicos subyacentes como por los mecanismos mediante los cuales favorece el desarrollo de enfermedades crónicas degenerativas ${ }^{14,15}$. Con la participación de los factores definidos, es difícil determinar los efectos independientes e interactivos del SMetab, en asociación a los que dependen de la enfermedad resultante, y cuya expresión además es distorsionada por las intervenciones terapéuticas y el tiempo de evolución. Por ejemplo, la inflamación es un mecanismo de daño crucial que se asocia a hiperinsulinemia y obesidad ${ }^{16}$, por lo que algunos proponen que sería parte integral del SMetab y uno de los mecanismos principales de progresión del daño, especialmente importante en la población más añosa ${ }^{17}$. El envejecimiento es uno de los principales factores de riesgo en muchas enfermedades crónicas, incluyendo los desórdenes cardiovasculares y neurodegenerativos. Por lo tanto, el SMetab y la inflamación podrían ser piezas de articulación central entre el envejecimiento y las enfermedades crónicas. 
Reconociendo en el SMetab una unidad patológica emergente, su estudio necesariamente implica disecar cada uno de sus factores constituyentes en cuanto a las modificaciones que llevan hacia diferentes condiciones patológicas y la forma como su existencia conjunta genera estas nuevas condiciones. La importancia conceptual del SMetab es, justamente, que su efecto en el individuo no corresponde a una simple suma de los distintos componentes, sino que emerge una identidad nueva como resultado de la combinación de las alteraciones de base. Como un ejemplo para visualizar la compleja interacción que se establece, podemos enumerar brevemente algunas de estas interacciones: la hiperglicemia genera múltiples cambios en la bioquímica celular, activándose vías de glucooxidación y la glicación no-enzimática de proteínas (asociado a la pérdida de función y agregación de las mismas) lo que lleva a la producción excesiva de radicales libres ${ }^{18,19}$. El estrés oxidativo a su vez resulta en daño oxidativo e inflamación ${ }^{16,20}$. La resistencia a la insulina se asocia a hiperglicemia, lo que junto a los efectos ya descritos favorece alteraciones metabólicas conducentes a la dislipidemia aterogénica característica de este síndrome ${ }^{21}$. La insulinoresistencia, como la dislipidemia, también induce sobreproducción de radicales de oxígeno (ROS) en respuesta al aumento de oxidación de ácidos grasos libres por la mitocondria. Por último, la hiperglicemia e hiperlipidemia inducen modificaciones en el tono vasomotor arterial, incrementando la reactividad vasoconstrictora, por un mecanismo que parece depender de receptores $\alpha$-adrenérgicos y que se asocia a una reducción de la biodisponibilidad de óxido nítrico $(\mathrm{ON})$, alterando a su vez la vasodilatación dependiente del endotelio. Además, los niveles elevados de ROS tienen múltiples efectos sobre canales de potasio, los que también inducen alteraciones en los mecanismos de vasodilatación ${ }^{22}$. Se desprende de esta descripción breve, que la coexistencia e interacción recíproca de estos mecanismos es lo que genera la condición de riesgo sinérgica tan característica del SMetab.

En esta revisión, discutimos la participación del SMetab en 5 cuadros patológicos, dislipidemia, hígado graso, litiasis biliar, aterosclerosis, y condiciones degenerativas como la enfermedad de Alzheimer (EA). Si bien el SMetab es reconocido como un agravante, evidencia reciente sugiere que también constituye un factor de riesgo importante por sí mismo, con una relación mecanicista causal. Sólo recientemente se han comenzado a dilucidar los mecanismos fisiopatológicos comunes que existen en estos cuadros, estudiando como interactúan y se influencian mutuamente. La ateroesclerosis se asocia a cambios en el metabolismo lipídico, inflamación e hipertensión. Estos mismos factores, junto con la hiperglicemia, están también involucrados en las enfermedades neurodegenerativas, ya sea a través de mecanismos directos o mediados por la isquemia cerebral ${ }^{14,15,23}$.

\section{Dislipidemia}

El patrón clásico de dislipidemia asociada al SMetab se caracteriza por un perfil proaterogénico constituido por hipertrigliceridemia, colesterol de lipoproteínas de alta densidad (HDL) bajo y niveles elevados de partículas de lipoproteínas de baja densidad (LDL) ${ }^{24}$. Su sustrato fisiopatológico es un aumento en el flujo de ácidos grasos libres desde el tejido adiposo hacia el hígado como consecuencia de la resistencia insulínica periférica, lo que conlleva a una mayor síntesis de triglicéridos y un aumento en la producción hepática de lipoproteínas de muy baja densidad (VLDL) ${ }^{24,25}$. Por otro lado, la resistencia a la insulina también induciría cambios en la actividad de las lipasas lipoproteica periférica y endotelial, contribuyendo adicionalmente al estado hipertrigliceridémico ${ }^{26,27}$. Independiente del mecanismo fisiopatológico subyacente, la presencia de hipertrigliceridemia es un muy buen indicador de resistencia a la insulina y un criterio para el diagnóstico del SMetab.

Otro trastorno lipídico importante del SMetab es la reducción en los niveles de colesterol HDL como consecuencia de la hipertrigliceridemia ${ }^{24}$. En presencia de un aumento en los triglicéridos plasmáticos transportados en las lipoproteínas no-HDL, se estimula la actividad de la enzima de transferencia de ésteres de colesterol, la cual intercambia triglicéridos de VLDL, lipoproteínas de densidad intermedia (IDL) y LDL por colesterol esterificado de $\mathrm{HDL}^{28}$. El enriquecimiento de las partículas de HDL con triglicéridos aumenta su susceptibilidad al efecto lipolítico de la lipasa hepática, acelerando el catabolismo de estas lipoproteínas $^{29}$.

Finalmente, si bien el SMetab no se asocia habitualmente a hipercolesterolemia LDL, la composición lipídica de las LDL también se modifica 
por el enriquecimiento relativo en triglicéridos en comparación a colesterol y fosfolípidos, lo que lleva, por remodelamiento lipolítico, a la aparición de partículas de LDL pequeñas y densas que tienen una mayor actividad aterogénica ${ }^{30}$.

\section{Hígado graso}

Entre las alteraciones fisiopatológicas asociadas al SMetab, la acumulación de grasa en el hígado ha concitado gran interés en las últimas dos décadas $^{31,32}$. Se ha reconocido este fenómeno como condicionante, en un subgrupo de individuos, de la ocurrencia de cirrosis hepática e incluso hepatocarcinoma $^{33}$. Dado que en la mayoría de los casos, los cambios histológicos hepáticos observados en individuos con SMetab son similares a los observados en sujetos que consumen crónicamente alcohol, pero también son detectados en individuos abstemios o con un consumo no significativo (menos de 20-30 g de alcohol al día), se ha acuñado el término hígado graso no alcohólico (HGNA) como descriptor de esta condición hepática ${ }^{32}$.

El HGNA comprende un espectro patológico amplio que incluye, como característica básica, la presencia de esteatosis predominantemente macrovesicular en al menos 5\% de los hepatocitos, asociada o no a cambios inflamatorios y fibrosis pericelular $y / 0$ sinusoidal ${ }^{34}$. La presencia de elementos histológicos de inflamación o fibrosis define la esteatohepatitis no alcohólica (EHNA), considerada una forma más agresiva de la enfermedad y cuya importancia radica en su potencial progresión a cirrosis hepática, la que ha sido estimada en $20 \%$ en dos décadas ${ }^{33}$.

El HGNA es considerado actualmente la enfermedad hepática más común. Su prevalencia estimada en la población general alcanza cifras entre 10 y $34 \%{ }^{35}$. Grupos seleccionados, como pacientes con obesidad mórbida o diabetes, exhiben frecuencias de hasta $90 \%{ }^{36,37}$. La variación observada en los estudios epidemiológicos se explica, entre otras razones, por factores de tipo étnico y por el tipo de herramienta diagnóstica empleada. Los estudios que han empleado técnicas de alta sensibilidad como la espectroscopía por resonancia magnética, como el Dallas Heart Study, han determinado una prevalencia de $33,6 \%{ }^{38}$. Tomados en su conjunto, la prevalencia de HGNA es tan significativa que podría aumentar la carga de enfermedad por hepatopatía crónica significativa en las próximas décadas. Más aún, su estrecha interrelación con el SMetab ha determinado que también se relacione al HGNA con un incremento en el riesgo cardiovascular ${ }^{39}$.

\section{Litiasis biliar}

La enfermedad litiásica biliar por cálculos de colesterol es un proceso de alta prevalencia y costo en países occidentales. Diferentes estudios epidemiológicos han identificado varios factores de riesgo, como el género femenino, la edad, la obesidad, la dislipidemia (especialmente el colesterol HDL bajo) y la hiperinsulinemia ${ }^{40-42}$. La asociación de la litiasis biliar con este conjunto de alteraciones metabólicas ha apoyado la hipótesis que esta enfermedad es otro cuadro característico del SMetab. De hecho, el aumento de la frecuencia en el SMetab y la resistencia insulínica se asocia con el aumento en la prevalencia de la litiasis biliar a nivel mundial ${ }^{43-45}$.

Actualmente se considera que la resistencia a la insulina sería el factor fisiopatológico común subyacente en la asociación entre litiasis biliar, diabetes mellitus y obesidad, y el postulado más aceptado es que el vínculo patogénico entre la resistencia a la insulina, obesidad y colelitiasis sería el aumento de la saturación biliar de colesterol derivada del aumento de síntesis hepática y de la hipersecreción de colesterol a la bilis ${ }^{44}$. Así, se plantea que un mayor flujo de ácidos grasos hacia el hígado favorecería el metabolismo hacia la formación de acetato, intermediario que constituye el sustrato inicial para la síntesis celular de colesterol. Por otra parte, el aumento de la expresión del transportador canalicular de colesterol ABCG5/ G8, parece ser un factor de riesgo primario para la hipersecreción de colesterol biliar en condiciones de resistencia a la insulina ${ }^{46}$. Sin embargo, se requieren estudios adicionales para comprender mejor los mecanismos de asociación fisiopatológica entre litiasis biliar de colesterol y SMetab desde un punto de vista metabólico, celular y molecular y la desregulación de la secreción de colesterol en esta condición metabólica.

\section{Ateroesclerosis}

Los sujetos que padecen de SMetab presentan un riesgo aumentado de desarrollar enfermedad cardiovascular ateroesclerótica, aunque este efecto 
parece ser más importante en hombres que en mujeres ${ }^{47}$. Esta asociación se basa en diferente tipo de evidencia que va desde estudios básicos y patológicos hasta trabajos clínicos, imageneológicos y epidemiológicos. La presencia de SMetab determina un riesgo estimado de eventos coronarios isquémicos de $10-20 \%$ en pacientes varones sin enfermedad cardiovascular conocida (individuos en categoría riesgo intermedio), mientras que sujetos con historia cardiovascular personal previa que padecen SMetab simultáneamente, se categorizan como riesgo cardiovascular alto a muy alto $^{2,48-49}$. Sin embargo, existen posiciones críticas que plantean que la inclusión del SMetab como tal, en la predicción del riesgo cardiovascular, no agrega nada adicional al poder predictivo de la incorporación individual de cada uno de los elementos definitorios del síndrome ${ }^{1}$.

Característicamente, el SMetab se presenta como una combinación de factores de riesgo patogénico, tales como disfunción endotelial, dislipemia aterogénica, hipertensión arterial, hiperglicemia, hipercoagubilidad y un estado proinflamatorio, todos los cuales promueven el desarrollo de enfermedad ateroesclerótica ${ }^{50}$. Sin embargo, la insulinoresistencia y el hiperinsulinismo del SMetab per se inducen cambios moleculares, bioquímicos y celulares que influencian directa o indirectamente el inicio, progresión y complicaciones de la ateroesclerosis. Por ejemplo, la resistencia a la insulina determina aumento de la expresión de moléculas de adhesión celular, con la consiguiente adherencia de linfocitos $\mathrm{T}$ al endotelio coronario y posterior migración hacia la íntima, además de la generación de un estado de procoagulación por incremento en la expresión del inhibidor del activador del plasminógeno 1 (PAI-1) y de inestabilidad de las placas de ateroma secundaria a mayor expresión de metaloproteinasas por los macrófagos ${ }^{51}$. Así, el trastorno metabólico subyacente favorece por si mismo la formación de la estría lipoidea, el crecimiento de la placa ateromatosa con su componente inflamatorio, y la ruptura que lleva a los eventos cardiovasculares isquémicos agudos.

\section{Enfermedad de Alzheimer}

La EA da cuenta de alrededor de $65 \%$ de las demencias y ha alcanzado dimensiones epidémicas en el mundo. Si bien la teoría más aceptada para la patogénesis de la EA asocia la agregación del $\mathrm{A} \beta$ y formación de placas seniles al proceso neurodegenerativo ${ }^{52}$, hallazgos recientes apoyan la asociación de la EA con mecanismos fisiopatológicos cerebrovasculares. De hecho, la enfermedad cerebrovascular (ECV) y la EA compartirían su asociación al SMetab como factor de riesgo ${ }^{53,54}$.

La asociación de la EA con el SMetab se apoya en evidencia epidemiológica, patológicas, de imageneología y clínica ${ }^{55,56}$. Sin embargo, debido a la complejidad de los mecanismos biológicos subyacentes a las enfermedades neurodegenerativas, y al hecho que su diagnóstico en general se realiza décadas después del inicio de los cambios patogénicos, varios aspectos de la interrelación entre SMetab y estos cuadros están aún en estudio, siendo necesaria investigación adicional para establecer la existencia de relaciones causales entre ellas. Por otro lado, si bien su asociación patogénica exacta no está claramente dilucidada, el SMetab parece inducir directamente un aumento en el depósito de $\mathrm{A} \beta$ amiloidogénico, como también la hiperfosforilación y agregación de tau, las dos lesiones anatomopatológicas características de la EA (Tabla 2). El SMetab tendría además un efecto cooperativo en la generación de condiciones de hipoperfusión cerebral y lesiones isquémicas como contribución principal al deterioro cognitivo y demencia tardía y/o favorecería la vulnerabilidad del cerebro a la disfunción neuronal y los procesos neurodegenerativos. Así, a través de los diversos mecanismos que parecen agruparse en torno a la resistencia a la insulina ${ }^{57}$, a largo plazo y eventualmente dependiendo de otras condiciones genéticas o ambientales, se desarrollaría la EA (Tabla 2).

\section{Tabla 2. Factores de riesgo de demencia asociados a la Resistencia a la Insulina*}

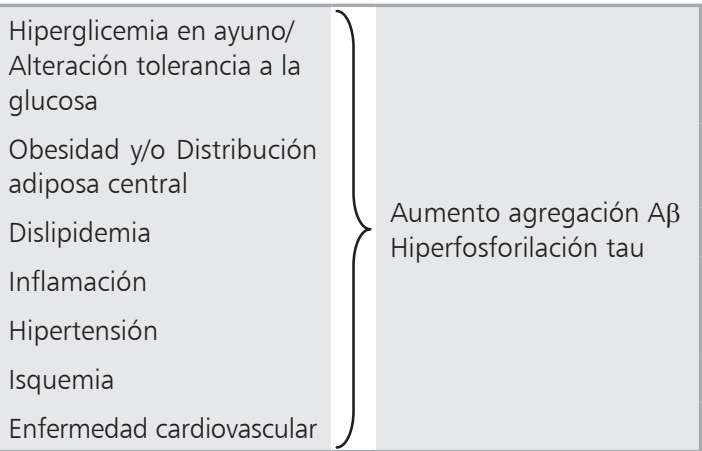

*Modificada de Craft., 2009 
Entre los mecanismos generales, las alteraciones hormonales inducidas por la obesidad central, que incluyen hipercortisolemia e hiperleptinemia contribuirían al déficit cognitivo produciendo alteraciones de memoria y aprendizaje. La diabetes puede favorecer la aparición de demencia a través de varios mecanismos, incluyendo complicaciones como hipertensión, enfermedad coronaria y renal, todos los cuales se asocian a disfunción cognitiva y EA. Además, la insulina es importante para los procesos cognitivos y tróficos neuronales y en condiciones de hiperinsulinemia crónica se observa una disminución de la insulina cerebra ${ }^{58}$. Por otro lado, la hiperinsulinemia se asocia a una mayor fosforilación de tau, componente principal de los ovillos neurofibrilares típicos de los cambios neurodegenerativos, y junto a la obesidad, a través de la disminución de los niveles de leptina, también aumenta el procesamiento amiloidogénico del A $\beta$. Por último, la obesidad se asocia con frecuencia a dislipidemia, la que presenta nexos muy complejos con la $\mathrm{EA}^{59}$.

Respecto a la hipertensión, entre los mecanismos posibles que se asocian a la EA podemos encontrar la ECV, infartos cerebrales silentes, atrofia cerebral, aterosclerosis en territorio cerebral, disfunción endotelial e hipo perfusión ${ }^{60}$.

\section{Importancia del síndrome metabólico para el manejo de las enfermedades crónicas}

Un manejo integral de las enfermedades crónicas requiere del manejo de los factores de riesgo subyacentes. De hecho, el manejo adecuado del SMetab modifica favorable y significativamente la evolución de cuadros patológicos crónicos asociados como diabetes y ateroesclerosis ${ }^{61}$. Además de la terapia farmacológica, incluyendo hipoglicemiantes, tratamientos antihipertensivos, drogas hipolipemiantes (las estatinas han demostrado ser especialmente beneficiosas), u otros, cada vez es más evidente que intervenciones en el estilo de vida (ejercicio, alimentación, tabaquismo, ingesta alcohólica) favorecen una disminución significativa de los factores de riesgo ${ }^{24,49,62}$, reduciendo las alteraciones cardio-metabólicas y el deterioro cognitivo. De hecho, el mejor camino para disminuir la incidencia de complicaciones, incluyendo las dos formas más comunes de demencia senil, la EA y la $\mathrm{ECV}^{24}$ parece ser la prevención del SMetab a través de cambios en el estilo de vida, y de nos ser estos suficientes, incorporar tratamiento farmacológico. Si bien en la actualidad el tratamiento farmacológico de un paciente con SMetab es similar al utilizado para tratar por separado los factores que confirman esta entidad nosológica, la comprensión de la fisiopatología del SMetab y sus mecanismos biológicos subyacentes, permitirá establecer las relaciones causales entre estos elementos, y desarrollar nuevos agentes terapéuticos que sobre múltiples mecanismos patogénicos, como serían drogas hipoglicemiantes capaces de antagonizar los efectos adversos del hiperinsulinismo como la adiponectina $\mathrm{a}^{63} \mathrm{o}$ estatinas que junto a la reducción del colesterol presentan efectos antiinflamatorios ${ }^{64}$, las cuales serían más efectivas para individuos con un SMetab, que la utilización de hipoglicemiantes clásicos carentes de estos efectos.

\section{Referencias}

1. Kahn R, Buse J, Ferrannini E, Stern M. The Metabolic Syndrome: Time for a Critical Appraisal. Joint statement from the American Diabetes Association and the European Association for the Study of Diabetes. Diabetes Care 2005; 28: 2289-304.

2. Grundy SM. Metabolic syndrome: a multiplex cardiovascular risk factor. J Clin Endocrinol Metab 2007; 92: 399-404.

3. International Diabetes Foundation Backgrounder 1: The IDF consensus worldwide definition of the metabolic syndrome. http://www.idf.org/webdata/docs/ IDF_Meta_def_final.pdf. [Consultado en octubre de 2008].

4. Alberti KG, Zimmet PZ. Definition, diagnosis and classification of diabetes mellitus and its complications. Part 1: Diagnosis and classification of diabetes mellitus provisional report of a WHO consultation. Diabet Med 1998; 15: 539-53.

5. Expert Panel on Detection, Evaluation, and Treatment of High Blood Cholesterol in Adults. Executive Summary of The Third Report on The National Cholesterol Education Program (NCEP) Expert Panel on Detection, Evaluation, and Treatment on High Blood Cholesterol in Adults (Adult Treatment Panel III). JAMA 2001; 285: 2486-97.

6. Alberti KG, Eckel RH, Grundy SM, Zimmet PZ, Cleeman JI, Donato KA, et al; International Diabetes Federation Task Force on Epidemiology and Prevention; National Heart, Lung, and Blood Institute; American Heart 
El síndrome metabólico: Factor de riesgo patogénico en diversas enfermedades crónicas - R. von Bernhardi et al

Association; World Heart Federation; International Atherosclerosis Society; International Association for the Study of Obesity. Harmonizing the metabolic syndrome: a joint interim statement of the International Diabetes Federation Task Force on Epidemiology and Prevention; National Heart, Lung, and Blood Institute; American Heart Association; World Heart Federation; International Atherosclerosis Society; and International Association for the Study of Obesity. Circulation 2009; 120: 1640-5.

7. Albala C, Vio F, Kain J, Uauy R. Nutrition transition in Chile: determinants and consequences. Public Health Nutrition 2002; 5: 123-8.

8. Alberti KG, Zimmet P, Shaw J. Metabolic syndrome-a new world-wide definition. A Consensus Statement from the International Diabetes Federation. Diabet Med 2006; 23: 469-80.

9. Valenzuela A, Olivos C, Rigotti A, Margozzini P, Olea R, Maiz A, et al. High level of agreement between the NCEP ATPIII and IDF metabolic syndrome definition in the Chilean general population: Results from the first National Health Survey 2003. Atherosclerosis Suppl 2007; 8: 121-2.

10. Wilson PWF, D'agostino RB, Parise H, Sullivan L, Meigs JB. Metabolic Syndrome as a Precursor of Cardiovascular Disease and Type 2 Diabetes Mellitus. Circulation 2005; 112: 3066-72.

11. Biessels GJ, Kapelle LJ. Increased risk of Alzheimer's disease in type II diabetes: insulin resistance of the brain or insulin-induced amyloid pathology? Biochem Soc Transac 2005; 33: 1041-4.

12. Margozzini P, Rigotti A, Ferreccio C, Quezada N, Garrido M, Valdés G. Hypertension and the cardiometabolic syndrome in Chile: a review of concepts and consequences for the developing world. Ther Adv Cardiovasc Dis 2007; 1: 83-90.

13. Martins IJ, Hone E, Foster JK, Sünram-Lea SI, Gnjec A, Fuller SJ, et al. Apolipoprotein E, cholesterol metabolism, diabetes, and the convergence of risk factors for Alzheimer's disease and cardiovascular disease. Mol Psychiatry 2006; 11: 721-36.

14. Duron E, Hanon O. Vascular risk factors, cognitive decline, and dementia. Vasc Health Risk Manag 2008; 4: 363-81.

15. Milionis HJ, Florentin M, Giannopoulos S. Metabolic syndrome and Alzheimer's disease: a link to a vascular hypothesis? CNS Spectr 2008; 13:606-613.

16. Esposito K, Nappo F, Marfella R, Giugliano G, Giugliano F, Ciotola, et al. Inflammatory cytokine concentrations are acutely increased by hyperglycemia in humans: role of oxidative stress. Circulation 2002; 106: 2067-72.
17. Helmersson J, Vessby B, Larsson A, Basu S. Association of Type 2 Diabetes With Cyclooxygenase-Mediated Inflammation and Oxidative Stress in an Elderly Population. Circulation 2004; 109: 1729-34.

18. Brownlee M. Biochemistry and molecular cell biology of diabetic complications. Nature 2001; 414: 813-20.

19. Brownlee M. The pathobiology of diabetic complications: a unifying mechanism. Diabetes 2005; 54: 1615 25.

20. Nishikawa T, Edelstein D, Du Xl, Yamagishi S, Matsumura T, Kaneda Y, et al. Normalizing mitochondrial superoxide production blocks three pathways of hyperglycaemic damage. Nature 2000; 404: 787-90

21. Nigro J, Osman N, Dart AM, Little PJ. Insulin resistance and atherosclerosis. Endocr Rev 2006; 27: 242-59.

22. Gutterman DD, Miura H, Liu Y. Redox modulation of vascular tone: focus of potassium channel mechanisms of dilation. Arterioscler Thromb Vasc Biol 2005; 25: 6718 .

23. Van Oijen M, De Jong FJ, Witteman JCM, Hofman A, Koudstaal PJ, Breteler MMB. Atherosclerosis and Risk for Dementia. Ann Neurol 2007; 61: 403-10.

24. Eckel RH, Grundy SM, Zimmet PZ. The metabolic syndrome. Lancet 2005; 365: 1415-28.

25. Raal FJ. Pathogenesis and management of the dyslipidemia of the metabolic syndrome. Metab Syndr Relat Disord 2009; 7: 83-8.

26. Blomhoff JP. Lipoproteins, lipases, and the metabolic cardiovascular syndrome. J Cardiovasc Pharmacol 1992; 20: S22-5.

27. Lamarche B, Paradis ME. Endothelial lipase and the metabolic syndrome. Curr Opin Lipidol 2007; 18: 298-303.

28. Rashid S, Uffelman KD, Lewis GF. The mechanism of HDL lowering in hypertriglyceridemic, insulin-resistant states. J Diabetes Complications 2002;16: 24-8.

29. Deeb SS, Zambon A, Carr MC, Ayyobi AF, Brunzell JD. Hepatic lipase and dyslipidemia: interactions among genetic variants, obesity, gender, and diet. J Lipid Res 2003; 44: 1279-86.

30. Rizzo M, Berneis K. Small, dense low-density-lipoproteins and the metabolic syndrome. Diabetes Metab Res Rev 2007; 23: 14-20.

31. Kotronen A, Yki-Jarvinen H. Fatty liver: a novel component of the metabolic syndrome. Arterioscler Thromb Vasc Biol 2008; 28: 27-38.

32. Kim CH, Younossi ZM. Nonalcoholic fatty liver disease: a manifestation of the metabolic syndrome. Cleve Clin J Med 2008; 75: 721-8.

33. Adams LA, Angulo P. Recent concepts in non-alcoholic fatty liver disease. Diabet Med 2005; 22: 1129-33.

34. Brunt EM. Pathology of fatty liver disease. Mod Pathol 
2007; 20 Suppl 1: S40-8.

35. Riquelme A, Arrese M, Soza A, Morales A, Baudrand R, Pérez-Ayuso RM, et al. Non-alcoholic fatty liver disease and its association with obesity, insulin resistance and increased serum levels of C-reactive protein in Hispanics. Liver Int 2008; 29: 82-8.

36. Boza C, Riquelme A, Ibáñez L, Duarte I, Norero E, Viviani $\mathrm{P}$, et al. Predictors of nonalcoholic steatohepatitis (NASH) in obese patients undergoing gastric bypass. Obes Surg 2005; 15: 1148-53.

37. Cusi K. Nonalcoholic fatty liver disease in type 2 diabetes mellitus. Curr Opin Endocrinol Diabetes Obes 2009; 16: 141-9.

38. Browning JD, Szczepaniak LS, Dobbins R, Nuremberg P, Horton JD, Cohen JC, et al. Prevalence of hepatic steatosis in an urban population in the United States: impact of ethnicity. Hepatology 2004; 40: 1387-95.

39. Edens MA, Kuipers F, Stolk RP. Non-alcoholic fatty liver disease is associated with cardiovascular disease risk markers. Obes Rev 2009; 10: 412-9.

40. Haffner SM, Diehl AK, Mitchell BD, Stern MP, Hazuda HP. Increased prevalence of clinical gallbladder disease in subjects with non insulin dependent diabetes mellitus. Am J Epidemiol 1990; 132: 327-35.

41. Attili AF, Capocaccia R, Carulli N, Festi D, Roda E, Barbara L, et al. Multicenter Italian Study on Epidemiology of Cholelithiasis. Factors associated with gallstone disease in the MICOL experience. Hepatology 1997; 26: 809-18.

42. Reaven GM. Banting lecture 1988: role of insulin resistance in human disease. Diabetes 1988; 37: 1595-607.

43. Nervi F, Miquel JF, Álvarez M, Ferreccio C, GarcíaZattera MJ, González R, et al. Gallbladder disease is associated with insulin resistance in a high risk Hispanic population. J Hepatol 2006; 45: 299-305.

44. Wang DQ, Cohen DE, Carey MC. Biliary lipids and gallstone formation. J Lipid Res 2009; 50: S406-11.

45. Tsai CJ, Leitzmann MF, Willett WC, Giovannucci EL. Macronutrients and insulin resistance in cholesterol gallstone disease. Am J Gastroenterol 2008; 103: 2932-9.

46. Biddinger SB, Haas JT, Yu BB, Bezy O, Jing E, Zhang W, et al. Hepatic insulin resistance directly promotes formation of cholesterol gallstones. Nat Med 2008; 14: 778-82.

47. Regitz-Zagrosek V, Lehmkuhl E, Weickert MO. Gender differences in the metabolic syndrome and their role for cardiovascular disease. Clin Res Cardiol 2006; 95: 13647.

48. Grundy SM, Cleeman JI, Merz CN, Brewer HB Jr, Clark LT, Hunninghake DB, et al. Coordinating Committee of the National Cholesterol Education Program. Implications of recent clinical trials for the National Cholesterol
Education Program Adult Treatment Panel III Guidelines. J Am Coll Cardiol 2004; 44: 720-32.

49. Grundy SM, Cleeman JI, Daniels SR, Donato KA, Eckel RH, Franklin BA, et al. Diagnosis and Management of the Metabolic Syndrome. An American Heart Association/National Heart, Lung, and Blood Institute Scientific Statement. Circulation 2005; 112: 2735-52.

50. Jiamsripong P, Mookadam M, Honda T, Khandheria BK, Mookadam F. The metabolic syndrome and cardiovascular disease: Part I. Prev Cardiol 2008; 11: 155-61.

51. Boyle PJ. Metabolic syndrome and macrovascular disease: mechanisms and mediators. Am J Med 2007; 120: S12-17.

52. Selkoe DJ. Alzheimer's disease: genes, proteins and therapy. Physiol Rev 2001; 81: 741-66.

53. Casserly I, Topol E. Convergence of atherosclerosis and Alzheimer's disease: Inflammation, cholesterol, and misfolded proteins. Lancet 2004; 363: 1139-46.

54. Román GC. Vascular dementia prevention: a risk factor analysis. Cerebrovasc Dis 2005; 20: 91-100.

55. Razay G, Vreugdenhil A, Wilcock G. The metabolic syndrome and Alzheimer disease. Arch Neurol 2007; 64: 93-6.

56. Vanhanen M, Koivisto K, Moilanen L, Helkala El, Hänninen $\mathrm{T}$, Soininen $\mathrm{H}$, et al. Association of metabolic syndrome with Alzheimer disease: a population-based study. Neurology 2006; 67: 843-7.

57. Craft S. The role of metabolic disorders in Alzheimer disease and vascular dementia. Arch Neurol 2009; 66: 300-5.

58. Erol A. An integrated and unifying hypothesis for the metabolic basis of sporadic Alzheimer's disease. J Alzheimer Dis 2008; 13: 241-53.

59. Wolozin B, Brown J, Theisler C, Silberman S. The cellular biochemistry of cholesterol and statins: insights into the pathophysiology and therapy of Alzheimer's disease. CNS Drug Rev 2004; 10: 127-46.

60. Whitmer RA, Sidney S, Selby J, Jonston SC, Yafte K. Midlife cardiovascular risk factors and risk of dementia in late life. Neurology 2005; 64: 277-81.

61. Early J. Comprehensive management of cardiometabolic risk factors. Clin Cornerstone 2007; 8: 69-80.

62. Pasinetti GM, Eberstein JA. Metabolic syndrome and the role of dietary lifestyles in Alzheimer's disease. J Neurochem 2008; 106: 1503-14.

63. Ziemke F, Mantzoros CS. Adiponectin in insulin resistance: lessons from translational research. Am J Clin Nutr 2010; 91: 258S-61S.

64. Khan T, Hamilton MP, Mundy DI, Chua SC, Scherer PE. Impact of Simvastatin on Adipose Tissue: Pleiotropic Effects in Vivo. Endocrinology 2009; 150: 5262-72. 